GuSTAVO MAXIMILANO DUTRA DA SILVA ${ }^{1}$

SÔNia MARIA Rolim ROSA LIMA ${ }^{2}$

José Cássio de Moraes ${ }^{3}$

Artigo Original

Palavras-chave

Sexualidade

Disfunção sexual

Menopausa

Síndrome X metabólica

Keywords

Sexuality

Sexual dysfunction

Menopause

Metabolic syndrome X

\section{Avaliação da função sexual em mulheres após a menopausa portadoras de síndrome metabólica}

\author{
Evaluation of sexual function in \\ postmenopause women with metabolic syndrome
}

\section{Resumo}

OBJETIVO: Avaliar a função sexual de mulheres após a menopausa com diagnóstico de síndrome metabólica. MÉTODOS: Estudo caso-controle, incluindo 195 mulheres após o período da menopausa lamenorreia $\geq 1$ ano, FSH $\geq 30 \mathrm{mUl} / \mathrm{mL}$ e idade entre 43 a 69 anos), atendidas no Departamento de Obstetrícia e Ginecologia da Faculdade de Ciências Médicas da Santa Casa de São Paulo e nas Unidades Básicas de Saúde do Programa de Saúde da Família da cidade de São Paulo. Foram coletados dados clínicos e avaliados o índice de massa corpórea e a circunferência abdominal. Na análise bioquímica foram solicitadas dosagens de colesterol total, HDL e LDL colesterol, triglicerídeos e glicemia de jejum. Foram consideradas com síndrome metabólica as mulheres que apresentaram três ou mais critérios diagnósticos: circunferência abdominal maior do que $88 \mathrm{~cm}$; triglicerídeos $\geq 150 \mathrm{mg} / \mathrm{dL}$; HDL colesterol $<50 \mathrm{mg} / \mathrm{dL}$; pressão arterial $\geq 130 / 85 \mathrm{mmHg}$ e glicemia de jejum $\geq 110 \mathrm{mg} / \mathrm{dL}$. As participantes foram divididas nos Grupos Controle (n=87) e Síndrome Metabólica (n=108). Empregou-se o questionário Female Sexual Function Index (FSFI) para avaliar a função sexual. RESULTADOS: A média de idade foi de 54,0 $\pm 4,7$ anos. $\bigcirc$ índice de disfunção sexual em mulheres com síndrome metabólica foi significativamente superior ao do Grupo Controle, quando considerado o FSFI <26,5 (90/108 [83,3\%] versus 42/87 [48,2\%], p<0,001) ou FSFI $\leq 23$ (62/108 $[57,4 \%]$ versus 16/87 [18,39\%], p<0,001). Os domínios desejo, excitação, lubrificação, orgasmo ( $p<0,001)$ e satisfação ( $p=0,002$ ) apresentaram escores inferiores nas mulheres portadoras da síndrome metabólica. Para o escore de dor não houve diferença significante $(p=0,57)$. Todos os componentes do diagnóstico da síndrome metabólica estiveram associados a maiores níveis de disfunção sexual (p<0,001). CONCLUSÃO: Mulheres após a menopausa com síndrome metabólica apresentam mais frequentemente disfunção sexual do que aquelas na mesma faixa etária que não são portadoras da síndrome.

\section{Abstract}

PURPOSE: To evaluate sexual function in postmenopausal women diagnosed with metabolic syndrome. METHODS: Case-control study with 195 postmenopausal women (amenorrhea $\geq 1$ year, $F S H \geq 30 \mathrm{mlU} / \mathrm{mL}$, aged 43 to 69 years) seen at the Department of Obstetrics and Gynecology, School of Medical Sciences of Santa Casa de São Paulo and in the Basic Units of the Family Health Program of São Paulo. Clinical data were collected, and body mass index and waist circumference were evaluated. Total cholesterol, HDL and LDL cholesterols, triglycerides, and fasting glucose were determined for biochemical analysis. We considered women to have the metabolic syndrome when they met three or more diagnostic criteria: waist circumference $>88 \mathrm{~cm}$ and triglycerides $\geq 150 \mathrm{mg} / \mathrm{dL}$; cholesterol $\mathrm{HDL}<50 \mathrm{mg} / \mathrm{dL}$; blood pressure $\geq 130 / 85 \mathrm{mmHg}$, and fasting glucose $\geq 110 \mathrm{mg} / \mathrm{dL}$. The participants were divided into Control Group ( $\mathrm{n}=87$ ) and Metabolic Syndrome Group ( $n=108)$. The Female Sexual Function Index (FSFI) was employed to assess the sexual function. RESULTS: Mean age was $54.0 \pm 4.7$ years. The rate of sexual dysfunction was significantly higher in women with the metabolic syndrome than in the Control Group, both when considering FSFI <26.5 (90/108 [83.3\%] versus 42/87 [48.2\%], $\mathrm{p}<0.0001)$ and FSFI $<23(62 / 108$ [57.4\%] versus 16/87 [18.39\%], $\mathrm{p}<0.001)$. The domains desire, arousal,
Correspondêncio Gustavo Maximiliano Dutra da Silva Departamento de Obstetrícia e Ginecologia Rua Doutor Cesário Motta Jr. 6 ] (EP: 01221-020 São Paulo (SP), Brasil

Recebido $07 / 03 / 2013$

Aceito com modificacōōes $29 / 07 / 2013$

\section{Trabalho realizado na Faculdade de Ciências Médicas da Santa Casa de São Paulo - FCMSCSP - São Paulo (SP), Brasil.} IPrograma de Pós-Graduação, Faculdade de Ciências Médicas da Santa Casa de São Paulo - FCMSCSP - São Paulo (SP); Universidade São Francisco campus de Bragança Paulista - Bragança Paulista (SP), Brasil.

2Departamento de Obstetrícia e Ginecologia, Faculdade de Ciências Médicas da Santa Casa de São Paulo - FCMSCSP - São Paulo (SP), Brasil.

${ }_{3}^{3}$ Departamento de Medicina Social, Faculdade de Ciências Médicas da Santa Casa de São Paulo - FCMSCSP - São Paulo (SP), Brasil. Conflito de interesses: não há. 
lubrication, orgasm $(p<0.001)$, and satisfaction ( $p=0.002)$ had lower scores in women with the metabolic syndrome. For the pain scores, there was no significant difference $(p=0.57)$ between groups. All components of the metabolic syndrome diagnosis were associated with higher levels of sexual dysfunction ( $p<0.001)$. CONCLUSION: Postmenopausal women with metabolic syndrome present more frequently sexual dysfunction than those of the same age who do not have it.

\section{Introdução}

A menopausa é um período de transição associado a ajustes hormonais, físicos, psicológicos e sociais ${ }^{1-3}$. Diversos estudos têm demonstrado o grande impacto do climatério na função sexual, podendo levar a quadros disfuncionais com alterações importantes no âmbito de saúde da mulher ${ }^{4-6}$. Assim, com a inclusão da satisfação sexual como condição sine qua non de saúde pela Organização Mundial de Saúde(OMS), temas que abordem este assunto estão em evidência.

A disfunção sexual (DS) é uma condição que pode afetar mulheres de diversas idades e raças ${ }^{7,8}$. É caracterizada por distúrbios e mudanças psicofisiológicas no ciclo da resposta sexual, incluindo distúrbios de desejo sexual, excitação, orgasmo e dor, sendo reconhecida como um problema difundido na sociedade influenciada tanto por fatores relacionados à saúde orgânica quanto psicossocial. Associa-se também com a piora da qualidade de vida, influenciando nos relacionamentos interpessoais 9 . No período do climatério, especialmente em mulheres após a menopausa, a prevalência da DS está em torno de 25 a $63 \%^{10-12}$

Entre os agravos à saúde que podem ocorrer neste período destacam-se a alta prevalência de hipertensão arterial, a intolerância à glicose, a obesidade e a hipercolesterolemia. Esses são os componentes para o diagnóstico da síndrome metabólica (SM), doença altamente presente na sociedade global atual e fator de risco para doenças cardiovasculares, as quais são responsáveis por altos níveis de mortalidade e diminuição da qualidade de vida ${ }^{13}$.

A SM é um transtorno complexo representado por um conjunto de fatores de risco cardiovascular, usualmente relacionados à deposição central de gordura e à resistência à insulina. Estudos em diferentes populações, como a mexicana, a norte-americana e a asiática, revelaram prevalências elevadas da SM, dependendo do critério utilizado e das características da população estudada, sendo que as taxas variavam de 12,4 a 28,5\% em homens e de 10,7 a $40,5 \%$ em mulheres ${ }^{14-16}$.

O estudo da SM tem sido dificultado pela ausência de consenso em sua definição e nos pontos de corte dos seus componentes, com repercussões na prática clínica e nas políticas de saúde. A terceira revisão dos especialistas em detecção, evolução e tratamento dos altos níveis de colesterol em adultos ${ }^{17}$, elaborada pelo Programa Nacional de Educação em Colesterol (NCEPs) é um documento baseado em evidências e extensivamente revisado com referências científicas que norteiam o assunto. O NCEP produz atualizações clínicas e novas orientações conforme os avanços científicos no manuseio do colesterol, entre outros.

A definição da OMS preconiza como ponto de partida a avaliação da resistência à insulina ou do distúrbio do metabolismo da glicose, o que dificulta sua utilização. Desse modo, na prática clínica, utilizam-se os critérios adotados pelo adult treatment panel (ATP) III ${ }^{18,19}$. Os critérios diagnósticos para SM no sexo feminino, de acordo com o ATP III, são: obesidade abdominal, definida como uma circunferência abdominal $(C A) \geq 88 \mathrm{~cm}$ (para mulheres); baixas concentrações séricas de HDL-colesterol ( $\leq 50 \mathrm{mg} / \mathrm{dL}$ para mulheres); hipertrigliceridemia, definida como triglicérides $\geq 150 \mathrm{mg} / \mathrm{dL}$; elevação dos níveis pressóricos a pelo menos 130/85 mmHg e níveis anormais de glicemia sérica, definidos como glicemia de jejum $\geq 110 \mathrm{mg} / \mathrm{dL}$. A SM representa a combinação de três componentes dentre os apresentados. Devido a sua simplicidade e praticidade, a definição recomendada pela I Diretriz Brasileira de Diagnóstico e Tratamento da SM foi adotada no presente trabalho ${ }^{19}$.

Diversos estudos indicam uma estreita relação entre a SM e a função sexual masculina prejudicada, mas existem poucos que fazem esta mesma relação entre as mulheres. $\mathrm{O}$ interesse no estudo da função sexual em mulheres com diagnóstico de SM é recente, e os estudiosos frisam a existência de poucos trabalhos que pleiteiem a sexualidade feminina e acreditam na necessidade de uma avaliação rotineira da função sexual em mulheres com SM, suas complicações, sua prevalência e seu impacto na qualidade de vida ${ }^{20-22}$.

Para avaliar a função sexual, diferentes questionários têm sido utilizados, sendo o Female Sexual Function Index (FSFI) considerado um instrumento de alto padrão e recomendado para uso generalizado na população feminina $^{23}$. O FSFI foi traduzido e validado para utilização em português ${ }^{24}$ e consta de um breve autorrelato, que avalia as dimensões-chave da função sexual na mulher, nas últimas quatro semanas.

No FSFI o ponto de corte de $\leq 26,5$ é atualmente aceito para o diagnóstico de DS em mulheres entre 18 e $74 \operatorname{anos}^{23}$. Entretanto, este índice pode fornecer taxas de DS variando de 22 a $50 \%$ em mulheres férteis, levando 
a uma possibilidade inadequada de que a maioria das mulheres férteis e saudáveis na quarta década de vida é portadora de $\mathrm{DS}^{25}$. Portanto, levantou-se a questão que o ideal seria considerar o escore total $\leq 23$. De fato, alguns autores têm considerado este dado: em um trabalho em que foram consideradas mulheres latinas na pré-menopausa sem uso de anticoncepcional oral, considerou-se escore do FSFI $\leq 23$ como ponto de corte para o diagnóstico das mulheres portadoras de $\mathrm{DS}^{25}$. Em mulheres após a menopausa também foi determinado como ponto de corte FSFI $\leq 23$, em um recente estudo que avaliou mulheres neste período portadoras da $\mathrm{SM}^{22}$. Dessa maneira, a exemplo de tais estudos e tendo em vista que também estava sendo avaliada uma população de mulheres de origem latina e após a menopausa, utilizou-se como ponto de corte $\leq 23$. Foi calculado também neste estudo os índices de DS, considerando o ponto de corte tradicional de FSFI $\leq 26,5$.

A ocorrência frequente das DS no climatério, a prevalência da SM nessa fase com seus diferentes componentes que poderiam interferir na resposta sexual, assim como os poucos trabalhos publicados sobre tal assunto, foram justificativas para o desenvolvimento do presente estudo. O objetivo principal foi avaliar a função sexual de uma amostra significativa de mulheres após a menopausa com SM e compará-las a um Grupo Controle não portador da síndrome.

\section{Métodos}

Trata-se de um estudo caso-controle. O grupo populacional foi constituído de mulheres após a menopausa que frequentam habitualmente o Ambulatório de Ginecologia Endócrina da Faculdade de Ciências Médicas da Santa Casa de São Paulo e as Unidades Básicas de Saúde Vila Barbosa, Vila Dionísia e Vila Santa Maria, pertencentes ao Programa de Saúde da Família da Coordenadoria Regional de Saúde Norte da Cidade de São Paulo, de fevereiro de 2011 a fevereiro de 2013.

O cálculo amostral foi embasado num estudo prévio que diagnosticou DS em torno de 39\% de uma população de 13.882 mulheres $^{11}$. Considerando tal prevalência, com nível de significância de 5\% e Odds Ratio=2, estimou-se a necessidade de avaliar, no mínimo, 144 mulheres. A pesquisa foi conduzida de acordo com a Declaração de Helsinque revisada em 2008. Todas as participantes assinaram o termo de consentimento livre e esclarecido, e o projeto foi aprovado pelo Comitê de Ética Médica da FCMSCSP projeto 077/11 e da Prefeitura de São Paulo CAAE 0016/12.

Foram consideradas mulheres após a menopausa aquelas com amenorreia $\geq$ um ano e FSH $\geq 30 \mathrm{mUI} / \mathrm{mL}$. O diagnóstico de SM foi determinado por meio das diretrizes do ATP III: CA $\geq 88 \mathrm{~cm}$; HDL-colesterol $(\leq 50 \mathrm{mg} / \mathrm{dL})$; triglicérides $\geq 150 \mathrm{mg} / \mathrm{dL}$; pressão arterial $\geq 130 / 85 \mathrm{mmHg}$ e glicemia de jejum $\geq 110 \mathrm{mg} / \mathrm{dL}$. Foram consideradas mulheres portadoras de SM aquelas com três componentes dentre os apresentados ${ }^{17}$.

A avaliação da função sexual foi realizada utilizando-se o $\mathrm{FFSFI}^{23}$, que é composto por 19 questões sobre cinco domínios da resposta sexual: desejo, excitação, lubrificação, orgasmo, satisfação e dor ou desconforto. As pontuações individuais são obtidas pela soma dos itens que compreendem cada domínio (escore simples), as quais são multiplicadas pelo fator de tal domínio e fornecem o escore ponderado. Portanto, quanto maior a pontuação, menor o risco de DS para um determinado domínio. O escore total pode variar de 2 a 36, sendo obtido pela soma daqueles ponderados de cada domínio, considerados como risco de DS os valores iguais ou menores que 26,5 pontos e em mulheres após a menopausa também considerado como ponto de corte FSFI $<23$. Foi utilizado o ponto de corte $\leq 23$, e calculou-se também o índice de DS considerando o ponto de corte de FSFI $\leq 26,5$.

As mulheres foram entrevistadas por um único investigador, o qual possuía um título reconhecido em sexualidade humana e experiência para realizar tal tarefa adequadamente. As pacientes que estavam no período após a menopausa e com vida sexual ativa foram incluídas no estudo e se submeteram à anamnese padronizada pelo nosso serviço.

A pressão arterial foi aferida no braço não dominante com esfigmomanômetro de mercúrio e precisão de $2 \mathrm{mmHg}$, com a mulher sentada e em repouso de, pelo menos, cinco minutos e após esvaziamento vesical. Duas medidas foram feitas por dois investigadores diferentes, previamente treinados, com um intervalo entre elas de aproximadamente 30 minutos. A pressão arterial foi obtida pela média das duas medidas. Braçadeira apropriada para obesas foi usada quando necessário. O peso corporal foi obtido em balança eletrônica (precisão de $0,1 \mathrm{~kg}$ ), com a bexiga vazia e a mulher vestindo apenas roupas íntimas. A estatura foi obtida em estadiômetro de parede com a mulher descalça e com precisão de $0,5 \mathrm{~cm}$. Desta forma, calculou-se o índice de massa corpórea (IMC = peso/estatura ${ }^{2}$ ), conforme recomendação da OMS para avaliar o estado nutricional. Para a medida da circunferência abdominal, a fita foi posicionada na menor curvatura localizada entre o último arco costal e a crista ilíaca. Todas as medidas antropométricas foram realizadas por um único pesquisador durante o projeto.

Em seguida, as participantes foram submetidas ao exame ginecológico e à coleta de citologia para exame de Papanicolaou. Foram também solicitados análises laboratoriais (colesterol total - CT e frações, triglicérides e glicemia de jejum), mamografia bilateral, ultrassonografia por via transvaginal, segundo propedêutica básica do serviço. Para os exames laboratoriais, as pacientes foram orientadas a manter um jejum de 12 horas. Todas as avaliações bioquímicas foram realizadas pelo Laboratório de Análises Clínicas da Faculdade de Ciências Médicas da Santa Casa de São Paulo. As mensurações de triglicerídeos, colesterol total, HDL e glicemia foram processadas pelo aparelho ADVIA 1650 da Multinacional 
Siemens Healthcare Diagnostics Inc. (Munich, Alemanha) pelo método colorimétrico de química seca. $\mathrm{O}$ método é linear até $800 \mathrm{mg} / \mathrm{dL}$ para triglicérides e $900 \mathrm{mg} / \mathrm{dL}$ para colesterol total. O LDL foi obtido subtraindo-se o valor do colesterol total, da soma do HDL e dos triglicerídeos, dividido por cinco, segundo fórmula de Friedwald.

Foram incluídas 272 mulheres; 160 provenientes do Ambulatório de Ginecologia Endócrina da Faculdade de Ciências Médicas da Santa Casa de São Paulo e 112 das Unidades Básicas de Saúde Vila Santa Maria, Vila Barbosa e Vila Dionísia. Após analisados os critérios de inclusão e exclusão, foram excluídas 77 , e as restantes divididas em dois grupos: Grupo Controle ( $\mathrm{n}=87$ ), com mulheres após a menopausa não portadoras de SM e Grupo SM ( $n=108$ ), com diagnóstico de SM.

Foram incluídas no estudo mulheres após a menopausa com vida sexual ativa, inclusive no mês precedente à entrevista; parceiro estável; portadoras de SM e não portadoras como controle. Foram excluídas pacientes com inatividade sexual; histórico de cirurgia pélvica e endometriose; sintomas do trato urinário baixo; uso de drogas ilícitas ou abuso de álcool; uso de drogas que comprovadamente provoquem DS (inibidores seletivos da recaptação de serotonina, neurolépticos); antecedentes pessoais de câncer, parceiro com DS, uso de terapia hormonal, portadoras de hipotireoidismo e doenças autoimunes.

Das pacientes excluídas: 1 relatou antecedente de câncer de mama, 2 foram diagnosticadas com lúpus eritematoso sistêmico, 5 haviam sido submetidas a cirurgias pélvicas anteriores por leiomiomatose e endometriose, 7 estavam em uso de terapia hormonal, 10 referiram parceiros sexuais com problemas que impediam o coito, 9 estavam na perimenopausa, 11 faziam uso de medicações para tratamento de hipertireoidismo, 12 faziam uso de fluoxetina para tratamento de depressão e 20 referiram não ter uma vida sexual ativa.

\section{Análise estatística}

As variáveis contínuas em ambos os grupos foram analisadas quanto à distribuição pelo teste de Shapiro-Wilk e descritas por meio de suas medianas, valores máximos e mínimos e comparadas pelo teste de Mann-Whitney. A comparação de escores nos dois grupos foi realizar pelo mesmo teste. As variáveis nominais (presença de DS e hipertensão) foram descritas por meio de suas frequências e comparadas pelo teste do $\chi^{2}$.

Foi utilizado o software SPSS 13.0 (SPSS Inc., Chicago, Illinois, USA) para as análises, tendo sido adotado um nível de significância de 5\%.

\section{Resultados}

As 108 mulheres com SM foram comparadas com 87 mulheres do Grupo Controle quanto à idade; ao tempo transcorrido após a menopausa; ao peso; ao IMC; à CA; ao CT; ao colesterol de alta densidade (HDL); aos triglicérides e à glicemia. Todos os componentes do diagnóstico de SM, inclusive a hipertensão arterial sistêmica, analisados separadamente pelo teste do $\chi^{2}$, estiveram associados a maiores níveis de DS, sendo encontradas diferenças significantes nos Grupos Controle e Estudo $(\mathrm{p}<0,001)$. Na Tabela 1 estão descritas as características clínicas, antropométricas e os parâmetros bioquímicos dos Grupos Controle e SM.

Analisando as características das mulheres portadoras de HAS quanto à presença ou ausência de DS, observouse que, no Grupo Controle, 20 são hipertensas $(22,9 \%)$, enquanto $16(18,3 \%)$ apresentam DS. No Grupo SM, 82 são hipertensas $(75,9 \%)$ e $62(57,4 \%)$ têm DS. Analisando comparativamente os grupos, foi verificado que houve diferença significante tanto em relação à HAS quanto à DS.

Após a aplicação do questionário FSFI, foram encontradas diferenças significantes entre as medianas dos escores de desejo, excitação, lubrificação, orgasmo e satisfação nos Grupos Controle e Estudo. Para o escore de dor não houve diferença significante. $\mathrm{Na}$ Tabela 2 estão descritos o escore total e por itens do FSFI.

Ao ser estudada a presença de DS usando como ponto de corte 23, observou-se que, no Grupo Controle, 18,4\% e, no Grupo SM, 57,4\% das mulheres apresentavam este distúrbio. Usando como ponto de corte 26,5 , foi notado $48,2 \%$ no Controle, e 83,3\% no SM foram diagnosticadas com DS.

Analisando nos dois grupos as mulheres com DS presente e relacionando-os com as variáveis HDL, triglicérides, glicemia, desejo, excitação, lubrificação, orgasmo, satisfação e dor, houve diferenças significantes para HDL, triglicérides, glicemia, lubrificação e dor (Tabela 3).

Tabela 1. Características clínicas e antropométricas e parâmetros bioquímicos dos Grupos Controle e Síndrome Metabólica, Faculdade de Ciências Médicas da Santa Casa de São Paulo, 2013

\begin{tabular}{lccccc}
\hline \multirow{2}{*}{ Características } & \multicolumn{2}{c}{ Grupo Controle } & \multicolumn{2}{c}{ Grupo SM } & \multirow{2}{*}{ Valor p } \\
\cline { 2 - 5 } & $(\mathrm{n}=87)$ & $($ min-máx) & $(\mathrm{n}=108)$ & (min-máx) & \\
\hline Iddade (anos) & 54 & $(46-66)$ & 53,5 & $(43-69)$ & 0,843 \\
TPM (anos) & 4 & $(1-10)$ & 4 & $(1-15)$ & 0,567 \\
Peso (kg) & 64 & $(45,3-90,6)$ & 71,9 & $(46,0-115,0)$ & $<0,001$ \\
IMC (kg/m²) & 25,3 & $(17,0-39,9)$ & 29,1 & $(19,0-49,3)$ & $<0,001$ \\
CA (cm) & 92 & $(61-117)$ & 100 & $(74-133)$ & $<0,001$ \\
CT (mg/dL) & 195 & $(143-286)$ & 229 & $(146-316)$ & $<0,001$ \\
HDL (mg/dL) & 53 & $(34-79)$ & 48 & $(29-78)$ & $<0,001$ \\
Triglicérides (mg/dL) & 114 & $(51-322)$ & 178 & $(52-433)$ & $<0,001$ \\
Glicemia (mg/dL) & 89 & $(73-138)$ & 103 & $(80-215)$ & $<0,001$ \\
\hline
\end{tabular}

TPM: tempo transcorrido após a menopausa; IMC: índice de massa corpórea; CA: circunferência abdominal; CT: colesterol total; HDL: colesterol de alta densidade; SM: Síndrome Metabólica; $p<0,001$ (Mann-Whitney). 
Tabela 2. Escore total do Female Sexual Function Index dos Grupos Controle e Síndrome Metabólica

\begin{tabular}{|c|c|c|c|c|c|}
\hline & \multicolumn{2}{|c|}{ Grupo Controle } & \multicolumn{2}{|c|}{ Grupo SM } & \multirow{2}{*}{ Valor $p$} \\
\hline & $(n=87)$ & (min-máx) & $(n=108)$ & (min-máx) & \\
\hline Desejo & 3,6 & $(1,2-6,0)$ & 2,4 & $(1,2-5,4)$ & $<0,001$ \\
\hline Excitação & 3,9 & $(2,1-6,0)$ & 3,3 & $(1,8-6,0)$ & $<0,001$ \\
\hline Lubrificação & 4,5 & $(1,8-6,0)$ & 3,4 & $(1,2-6,0)$ & $<0,001$ \\
\hline Orgasmo & 4,4 & $(1,2-6,0)$ & 3,6 & $(1,2-6,0)$ & $<0,001$ \\
\hline Satisfação & 4,8 & $(2,0-6,0)$ & 4 & $(2,0-6,0)$ & 0,002 \\
\hline Dor & 6 & $(2,0-6,0)$ & 6 & $(1,0-6,0)$ & 0,579 \\
\hline FSFI & 26,8 & $(13,9-34,8)$ & 22,4 & $(13,4-34,8)$ & $<0,001$ \\
\hline
\end{tabular}

Tabela 3. Mulheres com disfunção sexual presente dos Grupos Controle e Síndrome Metabólica com relação às medianas das variáveis colesterol de alta densidade, triglicérides, glicemia e escores do Female Sexual Function Index

\begin{tabular}{|c|c|c|c|c|c|}
\hline & \multicolumn{2}{|c|}{ Grupo Controle } & \multicolumn{2}{|c|}{ Grupo SM } & \multirow{2}{*}{ Valor $p$} \\
\hline & $(n=87)$ & (min-máx) & $(n=108)$ & (min-máx) & \\
\hline $\mathrm{HDL}(\mathrm{mg} / \mathrm{dL})$ & 54,5 & $(41-72)$ & 47,5 & $(32-78)$ & $<0,001$ \\
\hline Triglicérides (mg/dL) & 110,5 & $(56-322)$ & 182 & (52-324) & $<0,001$ \\
\hline Glicemia (mg/dL) & 89 & $(77-138)$ & 102 & $(80-215)$ & $<0,001$ \\
\hline Desejo & 2,4 & $(1,2-3,6)$ & 2,4 & $(1,2-4,2)$ & 0,527 \\
\hline Excitação & 2,7 & $(2,1-3,6)$ & 2,7 & $(1,8-4,2)$ & 0,905 \\
\hline Lubrificação & 3,3 & $(1,8-5,4)$ & 3 & $(1,2-4,2)$ & 0,016 \\
\hline Orgasmo & 3,6 & $(1,2-4,8)$ & 3,2 & $(1,2-4,8)$ & 0,289 \\
\hline Satisfação & 4 & $(2,0-5,2)$ & 4 & $(2,0-6,0)$ & 0,444 \\
\hline Dor & 3,6 & $(2,0-6,0)$ & 4,8 & $(1,0-6,0)$ & 0,016 \\
\hline
\end{tabular}

HDL: colesterol de alta densidade; SM: Síndrome Metabólica; $p<0,001$ (Mann-Whitney).

\section{Discussão}

Para que sejam realizadas aferições clínicas, como a função sexual, por exemplo, não há padrões físicos de validade. Portanto, neste estudo, optou-se pelo emprego de um instrumento validado para o português, o FSFI, que fornece resultados predominantemente qualitativos. Nesse sentido, apesar de sua natureza qualitativa e algumas limitações de generalização, foi importante a constatação da influência da SM na função sexual.

No presente estudo avaliou-se a prevalência de DS em dois grupos de mulheres após a menopausa com e sem diagnóstico de SM, definida de acordo com os critérios do NCEP-ATP III. Este é o primeiro trabalho na literatura nacional que avalia este tema.

A prevalência de DS foi maior no Grupo SM ao ser comparado com o Controle quando usados os pontos de corte de 23 e 26,5 ( 57,4 versus $18,4 \%$; FSFI $\leq 23$ e 83,3 versus $48,2 \%)$. Conforme proposto em trabalhos anteriores $^{8,22,25}$, julgamos que, na população brasileira, o ponte de corte menos rigoroso é mais adequado, pois percebe-se uma porcentagem muito alta de DS incompatível com o observado nas entrevistas. Também acredita-se serem necessários novos estudos para demonstrar o porquê na presente amostra, a exemplo dos estudos citados, termos um escore total FSFI abaixo do esperado. A possibilidade de variáveis socioculturais e religiosas, as quais são difíceis de ser mensuradas estatisticamente, é uma importante hipótese.

Cada item analisado pelo FSFI (desejo, excitação, lubrificação, orgasmo e satisfação) foi significativamente mais afetado nas mulheres do Grupo SM quando comparados ao Controle, exceto o escore de dor. Também foi encontrada diferença significante entre os dois grupos no critério índice de escore total. Tal achado pode se justificar pelo fato de que, em nossos critérios de inclusão, as mulheres com dor pélvica provenientes de endometriose e cirurgias pélvicas por miomatose (histerectomia abdominal) não foram incluídas. Outrossim, quando são analisadas, nos dois grupos, as mulheres com DS presente e relacionando-as à variável dor, houve diferença significante, demonstrando que mulheres após a menopausa realmente têm o quesito dor como um importante causador de DS.

Avaliando somente as mulheres disfuncionais também houve diferença significante em relação à lubrificação, demonstrando haver grande prejuízo nesse quesito nas mulheres portadoras de SM. Nos quesitos desejo, excitação $(p=0,9)$, orgasmo e satisfação, não houve diversidade significante. De qualquer maneira, sabe-se da dificuldade de avaliar separadamente esses quesitos, pois as diferenças são muito sutis.

A DS é uma desordem muito frequente e apresenta impacto importante na vida das mulheres após a menopausa. A prevalência desse distúrbio sofre grande variação, como demonstrado num estudo em que a prevalência foi de 26,7\% em mulheres na pré-menopausa e 52,4\% após a menopausa natural ${ }^{26}$. Essa variação pode ser atribuída a fatores como número de mulheres estudadas, desenhos de estudo, características sociodemográficas e questionários utilizados. Assim, a prevalência absoluta relatada deve ser tomada com cautela. $\mathrm{O}$ debate continua sobre qual melhor modelo representaria a resposta sexual feminina e quais os critérios que poderiam ser utilizados para definir a DS. Utilizou-se o questionário FSFI, que representa um método validado para avaliar a função sexual, no qual a angústia está correlacionada e incluída na definição da $\mathrm{DS}^{23}$. Além disso, este instrumento também é muito utilizado em estudos da literatura internacional semelhantes ao presente.

É interessante observar que, em um estudo em que foi também utilizado o questionário FSFI, mas em pacientes na pré-menopausa, o Grupo SM apresentou índice de pontuação menor que o Controle ${ }^{21}$. O diagnóstico de SM seguiu os critérios do ATPIII. Em outro estudo sobre o 
tema, foram avaliadas também mulheres na pré-menopausa com diagnóstico de SM, porém utilizando-se de um questionário desenvolvido pelo próprio autor, e foi observado que esta afecção constituiu um fator de risco independente para o componente desejo ${ }^{27}$. Vale lembrar que nesse trabalho o questionário utilizado diferiu-se do presente, e a SM foi diagnosticada com o uso dos critérios do International Diabetes Federation ${ }^{28}$.

Um estudo recente avaliou a função sexual de mulheres após a menopausa, sendo descrito 33\% de prevalência geral de DS no grupo de mulheres após a menopausa com SM comparado com $19 \%$ no Controle ${ }^{22}$. Os resultados deste estudo foram semelhantes, sendo o índice de DS em mulheres com SM significativamente superior ao do Grupo Controle. Estudos representativos, incluindo o Global Study of Sexual Attitudes and Behaviors and the National Social Life $e^{29}$, demonstraram que a DS está associada a problemas de saúde. Mudanças na função sexual podem ser um alerta ou uma consequência de importantes alterações, tais como diabetes mellitus, doenças metabólicas, doenças cardiovasculares, sintomas geniturinários ou câncer ${ }^{21}$.

Uma pesquisa em que o FSFI foi utilizado, não se observou diferença na pontuação média do domínio desejo do questionário ${ }^{22}$, resultado também notado neste trabalho. No entanto, tal como esperado para a mulher após a menopausa, a pontuação média FSFI para este domínio está abaixo do valor de corte de três para ambos os grupos, o que diferencia mulheres com distúrbio sexual hipoativo daquelas sem isso. É possível que ambos os grupos de mulheres apresentem redução do desejo sexual e que o instrumento não possa detectar diferenças sutis.

Analisando os parâmetros diagnósticos da SM (pressão arterial, hiperlipidemia, glicemia e obesidade), foram encontradas diferenças significantes quando comparados ao Grupo Controle em todos esses quesitos. $\mathrm{Na}$ literatura alguns itens foram descritos isoladamente como fatores de risco, porém no presente trabalho todos tiveram papéis importantes, entendendo ser a SM a principal preditora de DS ao ser comparada à qualquer componente da síndrome isolado.

Analisando o componente pressão arterial da SM, verificou-se maior prevalência de HAS em mulheres que apresentavam DS, com diferença significante. De fato, mulheres hipertensas relataram apresentar mais DS em comparação àquelas não hipertensas e recémdiagnosticadas com hipertensão ${ }^{30,31}$. Sabe-se que os trabalhos sobre o tratamento da hipertensão arterial e outras doenças crônicas negligenciam as mulheres e sua história sexual. Seria interessante avaliar também os antecedentes sexuais que precederam o uso da medicação anti-hipertensiva ou outros problemas que poderiam ser exacerbados com uso desses agentes.

Como exposto, a hiperlipidemia também foi preditora de DS. Sabemos que baixas concentrações séricas de HDL colesterol e aumento dos triglicérides constituem fatores de risco cardiovascular comuns e frequentemente encontrados em pacientes com SM. Tal enfermidade tem sido sugerida como a base do processo de desenvolvimento da DS, especialmente em mulher após a menopausa. Altas concentrações séricas de triglicérides apresentaram o dobro do risco de desenvolvimento da DS após a menopausa. Tais dados estão de acordo com os trabalhos prévios em mulheres, nas quais triglicérides foram fatores preditivos de menores escores do FSFI ${ }^{22,32}$. No entanto, em um estudo mais recente, a presença da SM foi o melhor preditor de DS comparado à qualquer componente da síndrome isolado, incluindo os triglicérides.

Nossos resultados demonstraram que tanto o peso, o IMC e a CA foram importantes promotores de menores escores da função sexual. Diferenças significantes foram encontradas com relação ao peso, à CA e ao IMC. Estudo recente demonstrou pela primeira vez que a obesidade afeta vários aspectos da função sexual, incluindo excitação, lubrificação, satisfação e orgasmo, mas não desejo e dor. Também ficou descrito que a ausência de relação entre IMC e FSFI em mulheres sem DS parece sugerir que obesidade pode ser um importante fator, mas deixa claro que estudos prospectivos devem ser realizados a fim de responder esta questão com mais clareza. Em relação à CA, também não foi mostrada correlação com o escore FSFI, sugerindo que a quantidade de gordura é mais importante do que sua distribuição ${ }^{25}$.

Outras características da SM, como a intolerância à glicose, resistência à insulina, e diabetes mellitus tipo 2, também têm sido associadas à $\mathrm{DS}^{33}$. A maioria dos estudos que até agora avaliaram DS em mulheres com diabetes mellitus tipo 2 possui um pequeno tamanho amostral $^{25}$. De qualquer maneira, reportam uma alta prevalência de DS em mulheres diabéticas comparadas às não diabéticas.

Em nosso estudo a glicemia afetou significantemente a função sexual, inclusive os domínios desejo, excitação, lubrificação, orgasmo e satisfação, exceto dor. Quando foram analisadas as mulheres com DS de ambos os grupos também notou-se relação significante quanto aos níveis de glicemia. Pesquisadores ${ }^{34}$ avaliaram 613 mulheres diabéticas e 524 não diabéticas na Jordânia, encontrando prevalência da DS de $59,6 \%$ em mulheres diabéticas com 50 ou mais anos, quando comparadas com $45,6 \%$ de não diabéticas da mesma faixa etária. Outro estudo ${ }^{35}$ descreveu um impacto negativo na sexualidade de mulheres após a 
menopausa com hiperglicemia, não se confirmando o mesmo em outros critérios da SM como hipercolesterolemia e HAS. Recentemente, demonstrou-se que mulheres com diabetes mellitus tipo 2 têm maior risco de DS; observou-se também que a hemoglobina glicada não se associou com risco de DS. Os fatores preditores independentes foram idade, SM e dislipidemia aterogênica e o protetor foi a prática frequente de atividade física ${ }^{25}$.
Algumas limitações de nossa pesquisa devem ser reconhecidas. A sua natureza transversal não nos permite inferir uma relação de causa e efeito, o pequeno número de mulheres e, como na maioria dos estudos epidemiológicos, há potencial para confusão devido à presença de covariáveis não controladas. Vale ressaltar que as mulheres foram recrutadas entre as que frequentam nosso ambulatório e não necessariamente representam a população em geral.

\section{Referências}

1. Dennerstein L, Koochaki P, Barton I, Graziottin A. Hypoactive sexual desire disorder in menopausal women: a survey of Western European women. J Sex Med. 2006;3(2):212-22.

2. Genazzani AR, Gambacciani M, Simoncini T. Menopause and aging, quality of life and sexuality. Climacteric. 2007;10(2):88-96.

3. Burger HG, Hale GE, Robertson DM, Dennerstein L. A review of hormonal changes during the menopausal transition: focus of findings from the Melbourne Women's Midlife Health Project. Hum Reprod Update. 2007;13(6):559-65.

4. Cabral PU, Canário AC, Spyrides MH, Uchôa SA, Eleutério Júnior J, Amaral RL, et al. Influence of menopausal symptoms on sexual function in middle-aged women. Rev Bras Ginecol Obstet. 2012;34(7):329-34.

5. Chedraui P, Pérez-López FR, Mezones-Holguin E, San Miguel G, Avila C; Collaborative Group for Research of the Climacteric in Latin America (REDLINC). Assessing predictors of sexual function in mid-aged sexually active women. Maturitas. $2011 ; 68(4): 387-90$.

6. Machado VS, Valadares AL, Costa-Paiva L, Morais SS, Pinto-Neto AM. Morbidity and associated factors in climacteric women: a population based study in women with 11 or more years of formal education. Rev Bras Ginecol Obstet. 2012;34(5):215-20.

7. Song $\mathrm{SH}$, Jeon $\mathrm{H}$, Kim SW, Paick JS, Son H. The prevalence and risk factors of female sexual dysfunction in young Korean women: an internet-based survey. J Sex Med. 2008;5(7):1694-701.

8. Aslan E, Beji NK, Gungor I, Kadioglu A, Dikencik BK. Prevalence and risk factors for low sexual function in women: a study of 1,009 women in an outpatient clinic of a university hospital in Istambul. J Sex Med. 2008;5(9):2044-52.

9. Basson R, Berman J, Burnett A, Derogatis L, Ferguson D, Fourcroy J, et al. Report of the international consensus development conference on female sexual dysfunction: definitions and classifications. J Urol. 2000; 163(3):888-93.

10. Hisasue S, Kumamoto $Y$, Sato $Y$, Masumori N, Horita H, Kato R, et al. Prevalence and female sexual dysfunction symptoms and its relationship to quality of life: a Japanese female cohort study. Urology. 2005;65(1):143-8.

11. Laumann EO, Paik A, Rosen RC. Sexual dysfunction in the United States: prevalence and predictors. JAMA. 1999;281(6):537-44.

12. Hayes RD, Dennerstein L, Bennett CM, Fairley CK. What is the "true" prevalence of female sexual dysfunction and does the way we assess these conditions have an impact? J Sex Med. $2008 ; 5(4): 777-87$.
13. Janssen I, Powell LH, Crawford S, Lasley B, Sutton-Tyrrell K. Menopause and the metabolic syndrome: the Study of Women's Health Across the Nation. Arch Intern Med. 2008; 168(14): 1568-75.

14. Aguilar-Salinas CA, Rojas R, Gómez-Pérez FJ, Valles V, Ríos-Torres $J M$, Franco $A$, et al. High prevalence of metabolic syndrome in Mexico. Arch Med Res. 2004;35(1):76-81.

15. Oh JY, Hong YS, Sung YA, Barrett-Connor E. Prevalence and factor analysis of metabolic syndrome in an urban Korean population. Diabetes Care. 2004;27(8):2027-32.

16. Ford ES, Li C, Zhao G. Prevalence and correlates of metabolic syndrome based on a harmonious definition among adults in the US. J Diabetes. 2010;2(3):180-93.

17. Expert Panel on Detection, Evaluation, and Treatment of High Blood Cholesterol in Adults. Executive summary of the Third report of The National Cholesterol Education Program (NCEP) Expert Panel on Detection, Evaluation, and Treatment of High Blood Cholesterol in Adults (Adult Treatment Panel III). JAMA. 2001 ;285(19):2486-97.

18. Grundy SM, Cleeman JI, Merz CN, Brewer HB Jr, Clark LT, Hunninghake DB, et al. Implications of recent trials for the National Cholesterol Education Program Adult Treatment Panel III guidelines. Circulation. 2004; 1 10(2):227-39.

19. Sociedade Brasileira de Cardiologia. I Diretriz Brasileira de Diagnóstico e Tratamento da Síndrome Metabólica. Arq Bras Cardiol. 2005;84 Supl 1:3-28.

20. Avis NE, Zhao X, Johannes CB, Ory M, Brockwell S, Greendale GA. Correlates of sexual function among multi-ethnic middle-aged women: results from the Study of Women's Health Across the Nation (SWAN). Menopause. 2005; 12:385-98.

21. Esposito K, Ciotola M, Marfella R, Di Tommaso D, Cobellis L, Giugliano D. The metabolic syndrome: a cause of sexual dysfunction in women. Int J Impot Res. 2005;17(3):224-6.

22. Martelli V, Valisella S, Moscatiello S, Matteucci C, Lantadilla C, Costantino A, et al. Prevalence of sexual dysfunction woman with and without metabolic syndrome. J Sex Med. 2012;9(2):434-41.

23. Rosen R, Brown C, Heiman J, Leiblum S, Meston C, Shabsigh R, et al. The Female Sexual Function Index (FSFI): a multidimensional self-report instrument for the assessment of female sexual function. J Sex Marital Ther. 2000;26(2):191-208.

24. Hentschel H, Alberton DL, Capp E, Goldim JR, Passos EP. Validação do Female Sexual Function Index (FSFI) para uso em língua portuguesa. Rev HCPA. 2007;27(1):10-4. 
25. Esposito K, Maiorino Ml, Bellastella G, Giugliano F, Romano M, Giugliano D. Determinants of female sexual dysfunction in type 2 diabetes. Int J Impot Res. 2010;22(3):179-84.

26. West SL, D'Aloisio AA, Agans RP, Kalsbeek WD, Borisov NN, Thorp JM. Prevalence of low sexual desire and hypoactive sexual desire disorder in a nationally representative sample of US women. Arch Intern Med. 2008;168(13):1441-9.

27. Ponholzer A, Temml C, Rauchenwald M, Marszalek M, Madersbacher $S$. Is the metabolic syndrome a risk factor for female sexual dysfunction in sexually active women? Int J Impot Res. 2008;20(1):100-4.

28. International Diabetes Federation. The IDF consensus worldwide definition of the metabolic syndrome [Internet]. Brussels: International Diabetes Federation; 2006 [cited 2012 Oct 6]. Available from: <http://www.idf.org/webdata/docs/IDF_Meta_def_final.pdf>

29. Laumann EO, Nicolosi A, Glasser DB, Paik A, Gingell C, Moreira $E$, et al. Sexual problems among women and men aged $40-80$ y: prevalence and correlates identified in the Global Study of Sexual Attitudes and Behaviors. Int J Impot Res. 2005; 17(1):39-57.
30. Okeahialam BN, Obeka NC. Sexual dysfunction in female hypertensives. J Natl Med Assoc. 2006;98(4):638-40.

31. Kütmeç C, Yurtsever S. Effects of sexual function of essential hypertensions in women. Eur J Cardiovasc Nurs. 201 1;10(1):56-63.

32. Esposito K, Ciotola M, Maiorino MI, Giugliano F, Autorino R, De Sio $M$, et al. Hyperlipidemia and sexual function in premenopausal women. J Sex Med. 2009;6:1696-703

33. Ogbera AO, Chinenye S, Akinlade A, Eregie A, Awobusuyi J. Frequency and correlates of sexual dysfunction in women with diabetes mellitus. J Sex Med. 2009;6(12):3401-6.

34. Abu Ali RM, Al Hajeri RM, Khader YS, Shegem NS, Ajlouni KM. Sexual dysfunction in Jordanian diabetic women. Diabetes Care. 2008;31(8):1580-1.

35. Chedraui P, Pérez-López FR, Blümel JE, Hidalgo L, Barriga J. Hyperglycemia in postmenopausal women screened for the metabolic syndrome is associated to increased sexual complaints. Gynecol Endocrinol. 2010;26:86-92. 